\title{
Altered static and dynamic functional network connectivity in post-traumatic headache
}

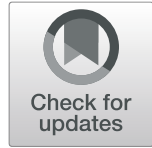

Fengfang $\mathrm{Li}^{1 \dagger}$, Liyan Lu${ }^{1 \dagger}$, Song'an Shang ${ }^{1}$, Huiyou Chen ${ }^{1}$, Peng Wang ${ }^{1}$, Vijaya Prakash Muthaiah², Xindao Yin ${ }^{1 *}$ and Yu-Chen Chen ${ }^{1 *}$

\begin{abstract}
Background: Post-traumatic headache (PTH) is a very common symptom following mild traumatic brain injury $(\mathrm{mTBI})$, yet much remains unknown about the underlying pathophysiological mechanisms of PTH. Neuroimaging studies suggest that aberrant functional network connectivity (FNC) may be an important factor in pain disorders. The present study aimed to investigate the functional characteristics of static FNC (sFNC) and dynamic FNC (dFNC) in $\mathrm{mTBI}$ patients with PTH.

Methods: With Institutional Review Board (IRB) approval, we prospectively recruited $50 \mathrm{mTBI}$ patients with PTH, who were diagnosed with ICHD-3 beta diagnostic criteria and $39 \mathrm{mTBI}$ without PTH who were well matched for age, gender and education. Resting-state functional magnetic resonance imaging (fMRI) scanning (3.0 T, Philips Medical Systems, Netherlands), Montreal Cognitive Assessment (MoCA) and headache symptom measurement (headache frequency and headache intensity) were performed. The resting-state fMRI sequence took 8 min and 10 s. Independent component analysis and sliding window method were applied to examine the FNC on the basis of nine resting-state networks, namely, default mode network (DMN), sensorimotor network (SMN), executive control network (ECN), auditory network (AuN), attention network (AN), salience network (SN), visual network (VN), and cerebellum network (CN). The differences in SFNC and dFNC were determined and correlated with clinical variables using Pearson rank correlation.

Results: For sFNC, compared with mTBI patients without PTH, mTB with PTH group showed four altered interactions, including decreased interactions in SN-SMN and VN-DMN pairs, increased sFNC in SN-ECN and SMNDMN pairs. For dFNC, significant group differences were found in State 2, including increased connectivity alteration in the DMN with CN, DMN with SMN, and AuN with CN. Significant reduced connectivity changes in the DMN with VN was found in State 4. Furthermore, the number of transitions $(r=0.394, p=0.005)$ between states was positively associated with headache frequency. Additionally, dwell time $(r=-0.320, p=0.025)$ in State 1 was negatively correlated with MoCA score.
\end{abstract}

\footnotetext{
*Correspondence: y.163yy@163.com; chenyuchen1989@126.com

'Department of Radiology, Nanjing First Hospital, Nanjing Medical University, No.68, Changle Road, 210006 Nanjing, China

Full list of author information is available at the end of the article
}

(c) The Author(s). 2021 Open Access This article is licensed under a Creative Commons Attribution 4.0 International License, which permits use, sharing, adaptation, distribution and reproduction in any medium or format, as long as you give appropriate credit to the original author(s) and the source, provide a link to the Creative Commons licence, and indicate if changes were made. The images or other third party material in this article are included in the article's Creative Commons licence, unless indicated otherwise in a credit line to the material. If material is not included in the article's Creative Commons licence and your intended use is not permitted by statutory regulation or exceeds the permitted use, you will need to obtain permission directly from the copyright holder. To view a copy of this licence, visit http://creativecommons.org/licenses/by/4.0/ The Creative Commons Public Domain Dedication waiver (http://creativecommons.org/publicdomain/zero/1.0/) applies to the data made available in this article, unless otherwise stated in a credit line to the data. 
Conclusions: MTBI patients with PTH are characterized with altered SFNC and AFNC, which could provide new perspective to understand the neuropathological mechanism underlying the PTH to determine more appropriate management, and may be a useful imaging biomarker for identifying and predicting mTBI with PTH.

Keywords: Post-traumatic headache, Mild traumatic brain injury, Functional magnetic resonance imaging, Functional network connectivity

\section{Introduction}

Post-traumatic headache (PTH) is a highly disabling secondary headache disorder and one of the most common sequelae of mild traumatic brain injury (mTBI)[1]. The prevalence of $\mathrm{PTH}$ is reported as high as $79 \%$ at 3 months and $65 \%$ at 12 months after mTBI[2]. PTH can be attributed to mTBI or moderate or severe traumatic brain injury, and PTH is often accompanied by mood, cognitive autonomic and sleep symptoms[3]. However, the underlying neuropathological mechanism of PTH after mTBI still remains unknown. Therefore, further work is needed to investigate the mechanisms of acute PTH and PTH persistence following mTBI, and to determine the specificity of the imaging findings for PTH.

Resting-state functional MRI was used to quantify brain functional connectivity (FC) and functional organization[4]. Previous studies have identified aberrant FC within networks including the default mode network(DMN), executive control network(ECN), salience network(SN) and visual networks(VN) in mTBI [5-7]. Furthermore, significant FC differences between migraine and mTBI with persistent PTH were found [810], and the altered functional network connectivity (FNC), such as DMN-attention network(AN) and VNAN, were identified in mTBI patients[11]. Overall, these results suggest that network alterations reflect clinically relevant phenomena in $\mathrm{mTBI}$ and $\mathrm{mTBI}$ with $\mathrm{PTH}$. However, most of the previous studies did not take into account important dynamic aspects that change over time.

Static FNC (sFNC) analysis ignores the fact that individual subjects may have slightly different psychological activities in different situations at different times[12]. In addition, emerging evidence indicates that the brain is a complex system with dynamic properties and timedependent [13]. Recently, studies have begun to take advantage of the powerful information contained in the temporal characteristics of the spontaneous FNC of the blood-oxygenation-level dependent (BOLD) signal [14]. Alterations in dynamic FNC(dFNC) are associated with specific psychiatric conditions, cognitive states, and neurological diseases[15-17]. In addition, studies of mTBI have demonstrated potential biomarker utility and the clinical relevance of dFNC[18, 19, 11]. However, alterations in dFNC are still largely unknown in mTBI patients with PTH.
The present study aimed to investigate the difference of sFNC and dFNC between the mTBI with and without $\mathrm{PTH}$, using resting-state $\mathrm{fMRI}$ and sliding-window analysis. We hypothesized that the sFNC, dFNC, and the temporal properties of dynamic FC states would characterize the underlying nature of $\mathrm{mTBI}$ patients with PTH.

\section{Materials and methods Participants}

This was a prospective study design and the Institutional Review Board (IRB) approval was obtained. A total of 89 mTBI patients participated in this study. MTBI was defined according to the American Congress of Rehabilitation Medicine[20]. Inclusion criteria were as follows: (a) patients aged 20 or older; (b) initial Glasgow Coma Score (GCS) of 13-15; (c) loss of consciousness $<30 \mathrm{~min}$; and (d) post-traumatic amnesia $<24 \mathrm{~h}$. Exclusion criteria were as follows: (a) a history of previous head injury; (b) history of pre-existing psychiatric or neurological disease; (c) history of illicit drug or alcohol abuse; (d) dental appliances that might distort the functional MR images; (e) history of migraine or any other headache prior to injury. and (f) MRI contraindications. Given the emergency care setting, it was not feasible to perform a full battery of cognitive assessments. Therefore, the clinical neurocognitive state of all participants of psychosis was quantified with the Montreal Cognitive Assessment (MoCA)[21], which is a sensitive cognitive screening test following $\mathrm{mTBI}$ and only requires limited training to administer. All subjects underwent the same MRI scan and cognitive function assessment within 0-7 days after trauma. In addition, none of the patients were receiving medication for headache. The present study was approved by the local ethics of Nanjing Medical University. Written informed consent were obtained from all participants before undergoing MRI.

\section{Headache symptom measurement}

After 12 months, all headache diagnoses were confirmed by two experienced headache specialists using International Classification of Headache Disorders 3rd edition, beta version (ICHD-3 beta) diagnostic criteria [22]. MTBI patients with PTH provided detailed information about the headache, including the main location, headache intensity, and headache frequency (day/month). All 
participants completed the visual analogue scale (VAS)[23], a numerical scale from 0 to 10 , with 0 representing no pain and 10 representing the most severe pain imaginable, to report the intensity of their headache.

\section{MRI data acquisition}

Images were acquired on $3.0 \mathrm{~T}$ Philips Ingenia scanner (Philips Medical Systems, Netherlands) using an 8channel head coil. The subjects were asked to lie quietly with their eyes closed, not to fall asleep, not to think about anything in particular, and to avoid any head movement during the scanning. Structural 3D T1weighted images were acquired with the three- dimensional turbo fast-echo (3D-TFE) T1WI sequence with the following specifications: repetition time (TR)/ echo time $(\mathrm{TE})=8.1 / 3.7 \mathrm{~ms}$; slices $=170$; gap $=0 \mathrm{~mm}$; thickness $=1 \mathrm{~mm} ; \mathrm{FA}=8^{\circ} ; \mathrm{FOV}=256 \mathrm{~mm} \times 256 \mathrm{~mm}$; and acquisition matrix $=256 \times 256$. Functional images were acquired using a gradient echo-planar imaging sequence with the following parameters: TR / TE $=2000 / 30 \mathrm{~ms}$; slices $=36$; gap $=0 \mathrm{~mm}$; thickness $=4 \mathrm{~mm}$; field of view $(\mathrm{FOV})=240 \mathrm{~mm} \times 240 \mathrm{~mm}$; acquisition matrix $=64 \times$ 64; and flip angle $(\mathrm{FA})=90^{\circ}$. A single resting-state fMRI run lasted for $8 \mathrm{~min}$ and $10 \mathrm{~s}$. The susceptibility weighted imaging (SWI) used a 3D gradient echo (GRE) sequence with the following parameters: $\mathrm{TR} / \mathrm{TE}=22$ $134 \mathrm{~mm} ; \mathrm{FA}=20$; slice thickness $=1 \mathrm{~mm}$; matrix $=$ $276 \times 319$; and FOV $=220 \mathrm{~mm} \times 220 \mathrm{~mm}$. The specifications for fluid-attenuated inversion recovery (FLAIR) were as follows: $\mathrm{TR} / \mathrm{TE}=7000 / 120 \mathrm{~ms}$; gap $=1.3 \mathrm{~mm}$; slices $=18$; slice thickness $=6 \mathrm{~mm} ; \mathrm{FA}=110^{\circ}$; and voxel size $=0.65 \times 0.95 \times 6 \mathrm{~mm}^{3}$. SWI and FLAIR are used to observe traumatic lesions. SWI showed low intensity and FLAIR showed high intensity in traumatic lesions.

\section{MRI data preprocessing}

Resting-state fMRI data preprocessing was performed using SPM12 software (http://www.fil.ion.ucl.ac.uk/spm/ ) implemented in MATLAB (version R2016b, MathWorks, Inc., Natick, MA, USA). The first ten scans were discarded to allow for magnetization equilibration, resulting in a total of 220 volumes. Resting-state data were realigned to the first volume to correct for interscan head motions; segmented into grey matter, cerebral spinal fluid, and white matter using the tissue probability maps; normalized into standard Montreal Neurological Institute template using nonlinear transformations and spatially smoothed with a Gaussian kernel of $6 \mathrm{~mm}$ fullwidth at half-maximum.

\section{Group ICA}

After data preprocessing, the data of all subjects were analyzed using the spatial independent component analysis method implemented by GIFT software[24, 25], and the data were decomposed into functional networks showing unique time history characteristics. The ICA analysis was performed in three stages: data reduction, application of the ICA algorithm, and back reconstruction for each individual subject. The number of independent components (ICs) was determined by using the minimum description length (MDL) criteria[26]. The data reduction was followed by a set of ICA, which was performed on the aggregate data of the subjects to produce an estimate of the ICA[24]. Then, the connectivity intensity value within each IC was converted into Zscore, reflecting the degree of correlation between the time series of a given voxel and the average time series of its corresponding components. To ensure the stability of the estimate, the algorithm was repeated 20 times in the ICASSO (http://research.ics.tkk.fi/ica/icasso/) algorithm, and the most central run was selected for further analysis. Spatiotemporal regression and regression reconstruction were used to obtain specific spatial maps and temporal processes of participants. Among the 34 components resulting from ICA, we selected 20 components (8 non-artifactual RSNs) as the focus of the subsequent analyses (Fig. 1) through visual inspection based on previous resting-state fMRI studies.

\section{SFNC analysis}

The sFNC analysis was performed using the MANCOVAN toolbox in GIFT software to explore changes in the predefined 20 spatial IC pairs of functional connections. First, at $0.01-0.15 \mathrm{~Hz}$, de-trend, de-peak and lowpass filtering were performed on the selected IC. Then, the pair correlations of these ICs were calculated and transformed using Fisher's Z-transform. In the general linear model, static FNC group difference estimation was performed for each pair of resting state networks, controlling for age and gender. After multiple comparison adjustments for FDR, the significance threshold was $P<0.05$.

\section{DFNC analysis}

To understand the dynamic nature of FNC, we used the temporal dFNC toolkit in GIFT software. In order to calculate the dFNC between ICA time processes, a sliding window method was used, in which the convolution of a rectangle of width 20 and TRs $=40 \mathrm{~s}$ with a Gaussian ( $\sigma=3$ TRs) was progressively refined, with each step advancing $1 \mathrm{TR}$ [27], resulting in $\mathrm{W}=128$ windows. The covariance between components was estimated according to the procedure outlined earlier[15]. Finally, the covariance matrices of each window were concatenated into a component $\times$ component $x$ window array to represent the change of covariance (correlation) between networks (components) over time. 


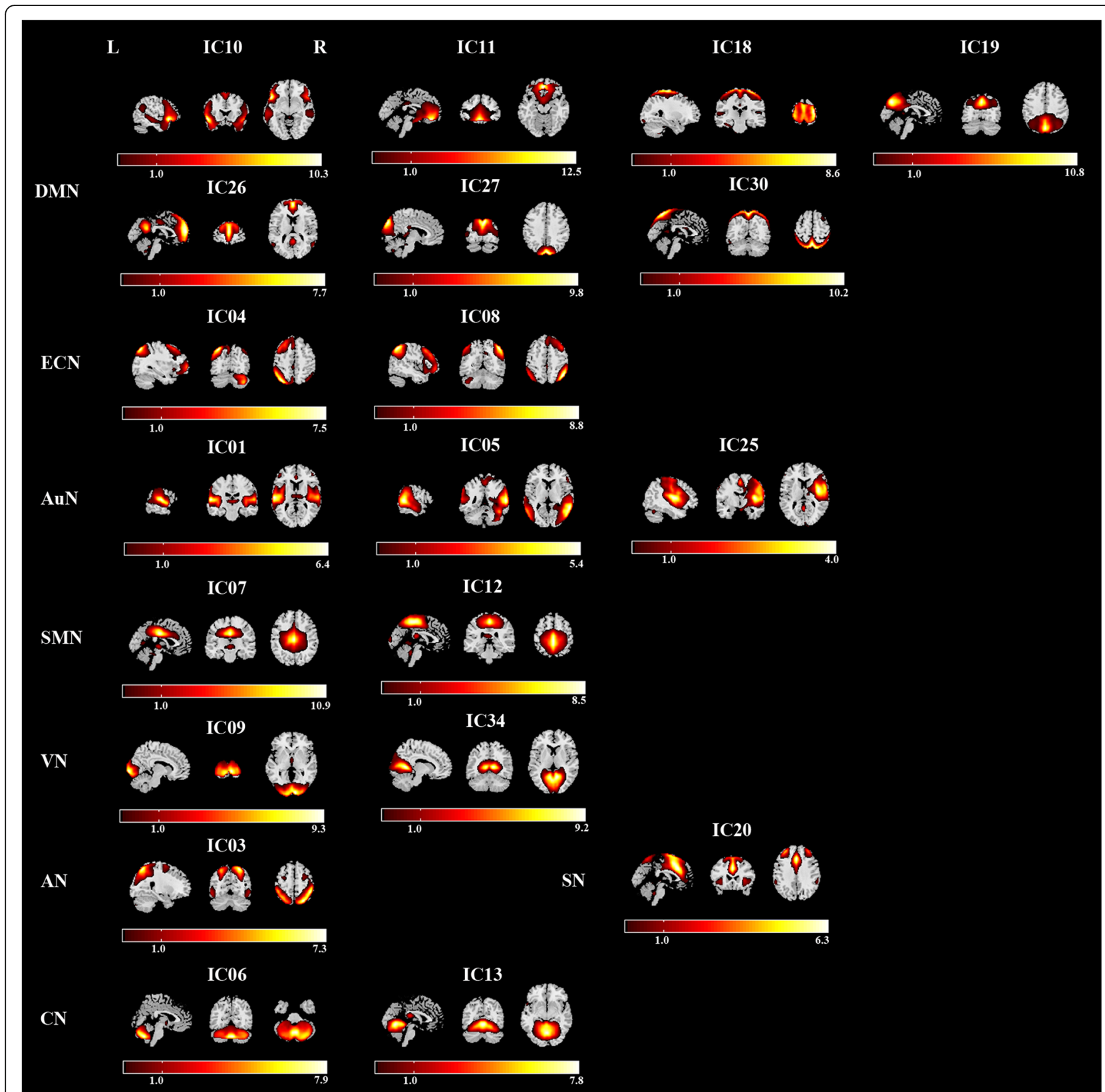

Fig. 1 Spatial maps (displayed at the three most informative slices) of 20 independent components (ICS) that have chosen as our networks of interest. R represent right and L represents left. DMN, default mode network; SMN, sensorimotor network; ECN, executive control network; AuN, auditory network; AN, attention network; SN, salience network; VN, visual network; CN, cerebellum network

The dFNC windows with 500 iterations and 150 repeats were divided into five clusters by using the $\mathrm{K}$ means clustering of square Euclidean distance realized by MATLAB [28]. The center of these clusters can be thought of as a small set of archetypes connecting "states"[29]. The number of optimal mental states was estimated using the elbow criterion (defined as the ratio of intra-cluster distance to inter-cluster distance). Using this method, $\mathrm{k}$ is 5 in the search window $\mathrm{k}$ is $2-10$ [15].
To check the structure of dFNC states between groups, we evaluated the group level dFNC states.

Differences between groups in dFNC and temporal properties

In each state differences between groups (mTBI+PTH and $\mathrm{mTBI}-\mathrm{PTH}$ ) in $\mathrm{dFNC}$ was investigated using two sample t-tests, and results were corrected for multiple comparisons using the false discovery rate (FDR) $(p<$ 
0.05). We analyzed the temporal properties of dFNC states by computing the fractional windows and average dwell time in each state, as well as the number of transitions between states. Mean "dwell" time is defined as the number of contiguous windows belonging to a state, the "fractional windows" is the total number of windows belonging to a state, and the "number of transitions" is defined as the number of transitions between states, indicating the reliability of each state. Two-sample $t$ test was used to analyze the significance of the mean residence time and transition times of each state in the mTBI + PTH group and the mTBI-PTH group $(p<0.05$, FDR corrected).

\section{Relationships between altered measurement and clinical variables in $\mathrm{mTBI}+\mathrm{PTH}$ group}

Pearson's correlation analysis was used to analyze the correlation between changed network attributes (network metrics and temporal attributes) and clinical variables including MoCA score, headache frequency, and headache intensity, controlling for age and gender. SPSS 19.0 (IBM Corporation, Armonk, NY, USA) was used for statistical analysis, and $P<0.05$ was used as the threshold.

\section{Results}

\section{Demographic and clinical characteristics}

The demographic and cognitive and headache characteristics of both mTBI patients with PTH group (mTBI+ PTH) and mTBI patients without PTH group (mTBI$\mathrm{PTH}$ ) were shown in Table 1 . There were no significant differences of age, gender, education, and MoCA performance between mTBI+PTH and mTBI-PTH group. Conventional imaging including SWI and FLAIR did not reveal any significant traumatic lesions.

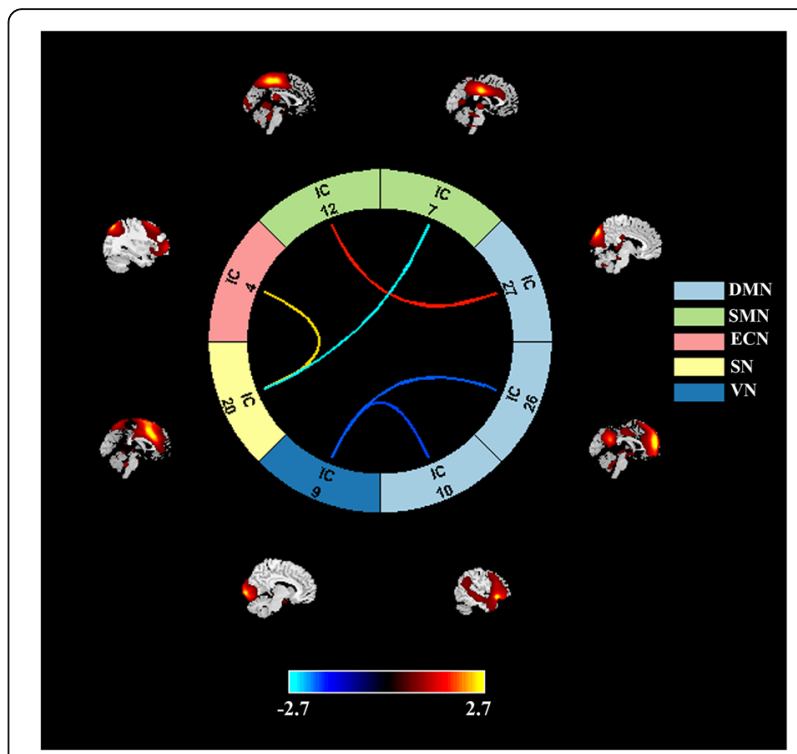

Fig. 2 Significant differences in the network connectivity in the default mode network (DMN), sensorimotor network (SMN), executive control network (ECN), salience network (SN), visual network (VN) between mTBI+PTH group and mTB-PTH group

\section{Networks of interests}

From a total of 34 components, 20 Independent components (IC) were chosen as our networks of interest (Fig. 1), which were grouped into the following eight networks: default mode network (DMN) (ICs10,11,18,19,26,27,30), sensorimotor network $(\mathrm{SMN})(\mathrm{ICs} 7,12)$, executive control network (ECN) (ICs4,8), auditory network (AuN) (ICs1,5,25), attention network (AN)(IC3), salience network (SN) (IC 20), visual network (VN) (ICs 9,34), cerebellum network (CN) (ICs $6,13)$.

Table 1 Demographic and cognitive variables of the mTBI with PTH patients and mTBI without PTH patients

\begin{tabular}{llll}
\hline Characteristics & mTBI+PTH $(\boldsymbol{n = 5 0 )}$ & mTBI-PTH $(\boldsymbol{n}=\mathbf{3 9})$ & $\boldsymbol{p}$-value \\
\hline Age (years) & $38.42 \pm 11.38$ & $42.36 \pm 10.75$ & 0.088 \\
Education (years) & $13.10 \pm 3.02$ & $12.49 \pm 3.08$ & 0.350 \\
Gender (Female/ Male) & $22 / 28$ & $15 / 24$ & 0.668 \\
MoCA scores & $24.12 \pm 2.68$ & $24.49 \pm 2.17$ & 0.489 \\
Headache characteristics & & & - \\
Predominant side & 19 & - & - \\
Right & 11 & - & - \\
Left & 20 & - & - \\
Bilateral & 30 & - & - \\
Unilateral & $11.74 \pm 7.18$ & - \\
Headache frequency & $4.66 \pm 1.94$ & - \\
Headache intensity & & \\
\hline
\end{tabular}



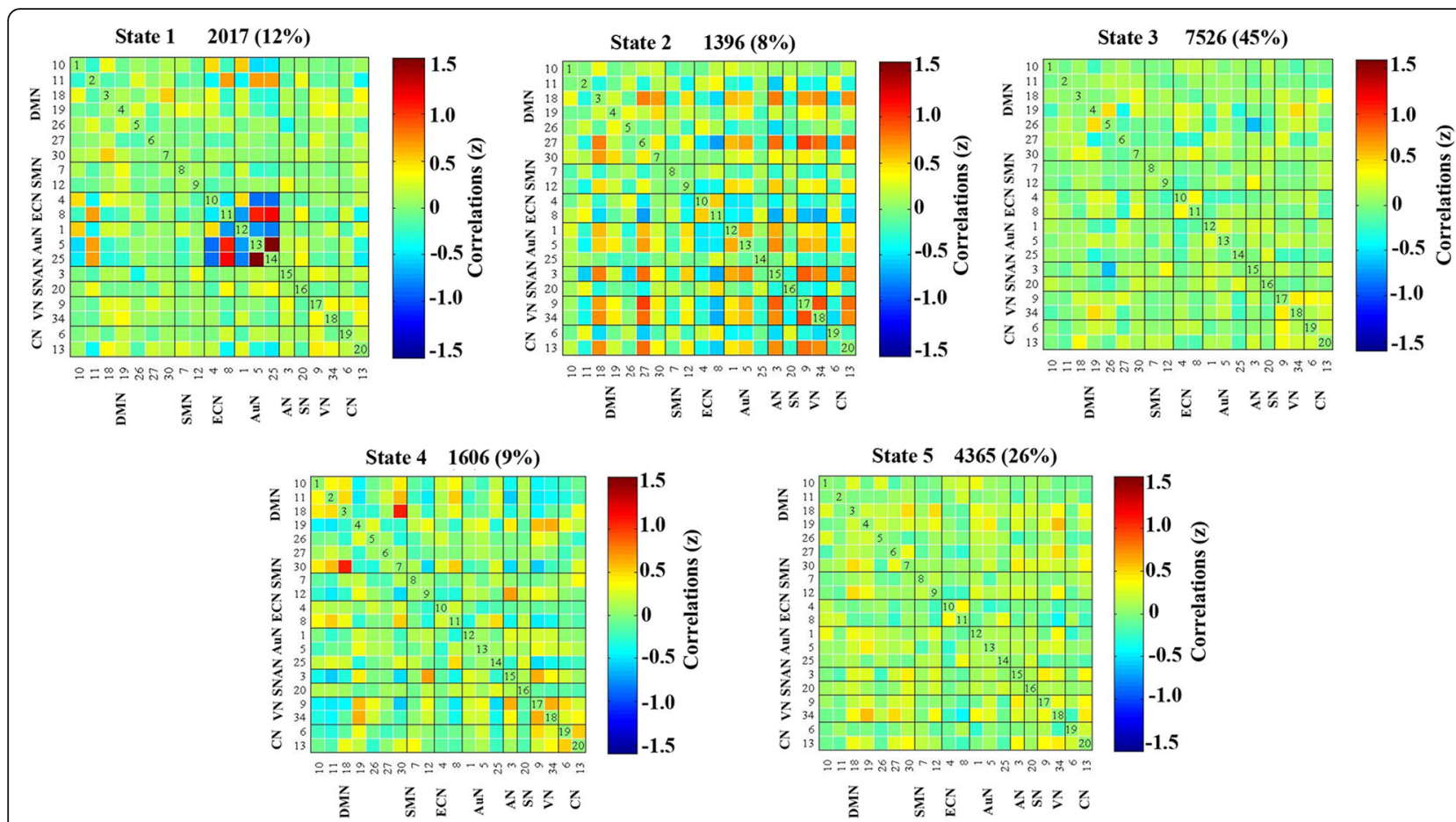

Fig. 3 Results of the clustering analysis per state. Cluster centroids for each state. The total number of occurrences and percentage of total occurrences are listed above each cluster median

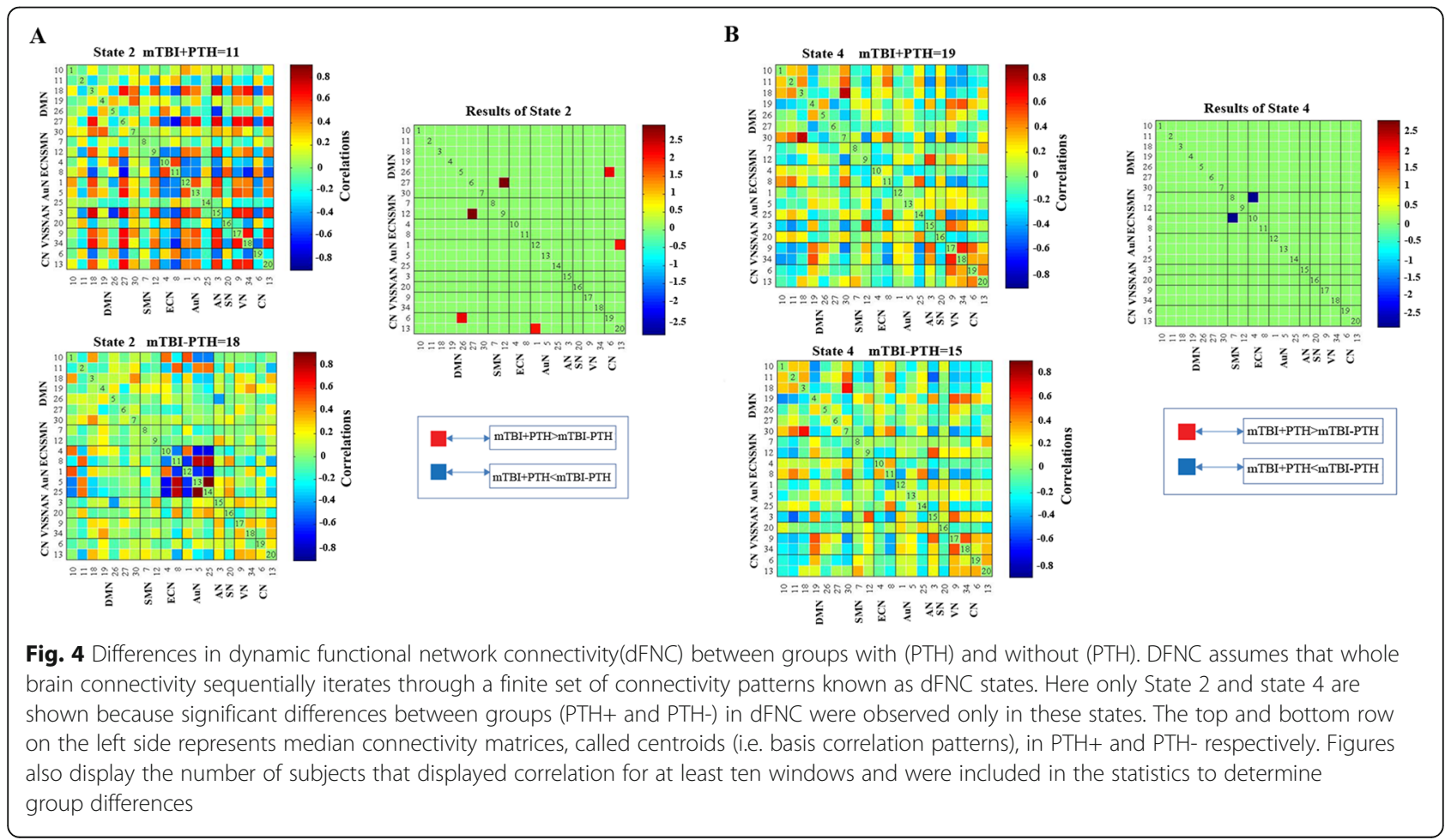




\section{Differences between groups in sFNC}

For the sFNC analysis, relative to the mTB-PTH group, the mTBI+PTH group exhibited significantly decreased interactions in two static connections, including the $\mathrm{SN}$ SMN connection and VN-DMN connection. Moreover, compared with the mTB-PTH group, the $\mathrm{mTB}+\mathrm{PTH}$ group also showed increased static FNC in the two interactions, including the SN-ECN and SMN-DMN. (Figure 2).

\section{Differences between groups in dFNC}

Using the k-means clustering method, we derived five highly structured states of FC that occurred repeatedly between individual scans and subjects. Figure 3 shows the five common functional connection states and corresponding cluster centers: the total percentages of these five states in all subjects were different, with State 1 $(12 \%)$, State $2(8 \%)$, State $3(45 \%)$, State 4 (9\%), State $5(26 \%)$. Significant differences between groups in dFNC were observed only in state 2 and states 4 (Fig. 4). Connectivity among six networks differed between groups in state 2, two represented DMNs (IC26 and IC27); Two represented CN (IC6 and IC13); The other two networks represented AuN (IC1) and SMN (IC12) respectively. Three sets of coupling (DMN-SMN, DMN-CN, AuN$\mathrm{CN})$ differed between mTBI+PTH and mTBI-PTH group (mTBI+PTH $>$ mTBI-PTH, $p<0.05$, FDR correction) (Fig. $4 \mathrm{~A})$. In state 4 , the between-network connections between DMN (IC26) and VN (IC9) differed between $\mathrm{mTBI}+\mathrm{PTH}$ group and $\mathrm{mTBI}-\mathrm{PTH}$ group (mTBI+PTH $<$ mTBI-PTH, $p<0.05$, FDR correction) (Fig. 4B).

\section{Temporal properties of FC states}

As shown in Fig. 5, significant group differences were identified in the mean dwell time of two states. Specifically, the mean dwell time in state 1 was significantly shorter in mTBI+PTH group compared to mTBI-PTH group (mTBI+PTH: 9.13 $\pm 15.58 ; \quad$ mTBI-PTH: $24.83 \pm$ $34.09, p<0.05)$. In contrast, the mean dwell time in state 4 was significantly longer in $\mathrm{mTBI}+\mathrm{PTH}$ group compared with mTBI-PTH group (mTBI +PTH: 14.91 21.93; mTBI-PTH:5.96 $\pm 11.30, p<0.05)$. In addition, the number of transitions between states in $\mathrm{mTBI}+\mathrm{PTH}$ group was smaller than in mTBI-PTH group (mTBI+ PTH: $2.54 \pm 2.22$; mTBI-PTH: $3.64 \pm 2.46, p<0.05$ ). However, we did not find any significant group differences in fractional windows in each state (all $p>0.05$ ).

\section{Correlation results}

The correlation between FC attributes and cognitive performance and headache measurements in the mTBI + PTH group was further analyzed (Fig. 5). We found that the number of transitions between states was positively associated with headache frequency $(r=0.394, p=$ 0.005), indicating the relationship between dynamic states changes and mTBI patients' headache frequency.

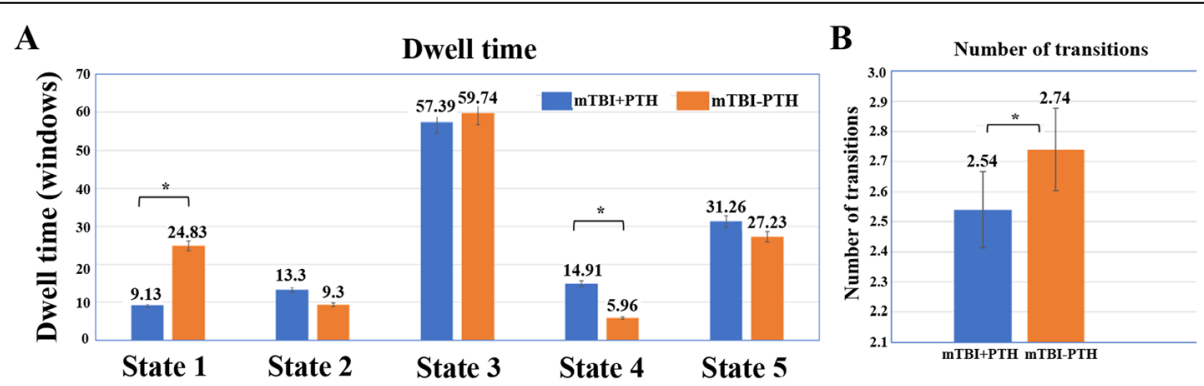

C

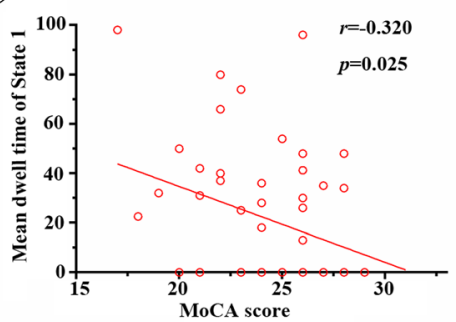

D

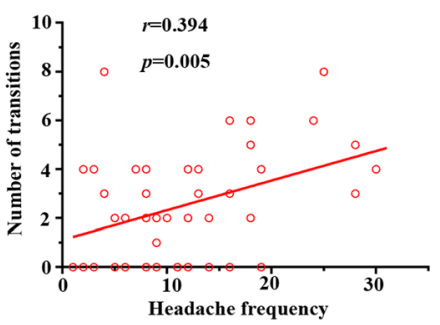

Fig. 5 Temporal properties of functional connectivity state analysis and correlation of clinical variables with temporal properties for mTBI with PTH patients. A mean dwell time (i.e. number of consecutive windows spent in each state before switching) and (B) number of transitions (i.e. switching between states) is depicted for mTBI with PTH and mTBI without PTH with error bars. Asterisk represent a significant group difference $(p<0.05$, FDR corrected). $\mathbf{C}$ the mean dwell time of state 1 was negatively correlated with MoCA score. $\mathbf{D}$ the number of state transitions was positively associated with the headache frequency in mTBI with PTH patients (all $p<0.05$, FDR corrected) 
Additionally, dwell time in State 1 was negatively correlated with MoCA score $(r=-0.320, p=0.025)$.

\section{Discussion}

The present study combined sFNC and dFNC analyses to investigate the whole brain features of mTBI with PTH with a focus on the FNC states as well as the temporal properties. Our results revealed the sFNC and $\mathrm{dFNC}$ features and altered dynamic temporal properties, moreover, the temporal characteristics is associated with the headache measurements and cognitive performance in $\mathrm{mTBI}+\mathrm{PTH}$ group.

For sFNC, our results showed that abnormal interactions of DMN-VN, DMN-SMN in mTBI with PTH patients, which are partly in line with prior neuroimaging results in mTBI with PTH and migraine patients $[8,30]$. The particular focus of mTBI patients on self-related symptoms, known as $\mathrm{PTH}$, may uniquely affect brain networks involved in pain perception and regulation. Notably, attention to pain or pain diversion has emerged as an important cognitive regulatory mechanism to explain pain perception in acute and chronic diseases[31]. We hypothesize that the association between cognitive control of the higher order cortex and hypersensitivity to pain sensation may be disrupted and may contribute to PTH after mTBI. Despite the similarity, mTBI with PTH patients also showed specific abnormalities in sFNC, such as decreased FNC in SN-SMN, and increased FNC in SN-ECN pairs. Our result provided further evidence that the insula, a main area belonging to the $\mathrm{SN}$, plays a specific role in pain regulation and emotional experience from information about body states[32]. Moreover, the pain can activate both the insula and the medial cingulate cortex whether acute or chronic, psychological or physical [33].

In comparing dFNC differences within RSNs between groups, we found significant between-group FNC differences among several RSNs, including the DMN, SMN, $\mathrm{CN}, \mathrm{AuN}$ and VN. Of note, the disrupted FNC in state 2 and state 4 was primarily related to the DMN, which is considered to be an endogenous neural network specialized for self-referential thinking and introspection [34]. Several studies on chronic pain have shown both increased and decreased cortical activity in brain regions within the DMN, and dysregulation of this network is thought to represent neuroplasticity in the brain during nerve repair and recovery after injury [35-37]. In line with these reports, we found aberrant $\mathrm{dFNC}$ between the DMN and other networks in mTBI+PTH group. Disrupted dFNC between the DMN and other networks could serve as a potential biomarker for neurocognitive dysfunction and PTH after mTBI. Additionally, recent researches have shown that the cerebellum is also involved in a variety of functions, including cognitive and affective processing, pain-related processing, athleticrelated processing, and thirsty experience[38, 39]. Similarly, our results showed disrupted dFNC between $\mathrm{CN}$ and DMN as well as AuN, suggesting that the PTH after mTBI may be related with the disruption of $\mathrm{CN}$. Therefore, we hypothesize that the association between hypersensitivity to pain and higher-order cortical cognitive control may be disrupted and as a whole involved in PTH following mTBI, and the results of FNC improve our understanding of the pathophysiological mechanisms of PTH in mTBI patients.

MTBI patients with PTH have significantly different dFNC temporal properties overall. The former dwelled different in the state 1 and state 4 , and showed a lower number of transitions to the strongly interconnected states. Thus, the reduced transition between brain states may be used for differentiating mTBI with PTH from mTBI without PTH. In addition, the temporal properties of dFNC are linked to cognitive outcome in multiple domains, such as memory, attention, execution and visuospatial function [16]. Zou et al. conducted a correlation analysis between dynamic temporal properties and clinical characteristics of headache, and observed a positive correlation between the changes in transition times between different states and the headache severity of chronic migraine[37]. Anyway, these observations may indicate the vulnerability of resting-state functional networks in mTBI with PTH, as well as emphasize the importance of exploring PTH after mTBI for the temporal dynamic FC. Therefore, the temporal properties of dynamic FC might help elucidate the neuropathological basis of the PTH, and serve as a potential imaging biomarker for investigating and predicting the PTH. Furthermore, the current findings might help clinicians to make therapeutic strategy for PTH.

There are some limitations in the current study. First, this is a preliminary cross-sectional study of dFNC changes in PTH after mTBI, and the sample size is limited, making it difficult to directly infer a causal relationship between the brain functional network of PTH status and cognitive impairment after mTBI. Longitudinal studies with larger samples are required. Second, this study only includes mTBI patients with and without PTH, but not taking health controls into consideration. Future studies including these three groups will be needed. Third, it has been suggested that dFNC analysis should be performed after at least $10 \mathrm{~min}$ of resting state acquisition[40]. Our resting state acquisition time was $8 \mathrm{~min}$, which allowed stable resting-state fMRI data to be obtained. Moreover, the time between injury and MRI scanning is not consistent, which may have effect on the results. Finally, there was no scale measuring neuropsychological states, including depression and anxiety during headaches, therefore, the influence of these 
neuropsychological factors on FNC has not been assessed.

\section{Conclusions}

This study explored for the static and dynamic FNC patterns in mTBI patients with PTH. Compared to mTBI patients without $\mathrm{PTH}$, the temporal properties of functional dynamics (number of transitions and dwelling time) were altered in mTBI patients with PTH, which were correlated with cognitive performance and headache characteristics. Taken together, the FNC and the temporal properties of dynamic FC might help elucidate the neuropathological basis of the $\mathrm{PTH}$, and serve as a potential imaging biomarker for investigating and predicting the PTH to determine more appropriate management.

\begin{abstract}
Abbreviations
fMRI: functional magnetic resonance imaging; PTH: post-traumatic headache; mTBI: mild traumatic brain injury; FC: functional connectivity; SFNC: static functional network connectivity; dFNC: dynamic functional network connectivity; DMN: default mode network; CN: cerebellum network; SMN: sensorimotor network; AuN: auditory network; VN: visual network; AN: attention network; ECN: executive control network; GCS: Glasgow Coma Score; MoCA: Montreal Cognitive Assessment; VAS: visual analogue scale; MDL: minimum description length
\end{abstract}

\section{Acknowledgements}

We thank all the patients and controls for their participation in the study.

\section{Authors' contributions}

$F L$ and $L L$ designed the study, performed the experiments, and wrote the manuscript. SS, HC, PW and VPM performed the experiments and analyzed the data. YX and Y-CC did the financial support, review, and final approval of the paper to be published. All authors read and approved the final manuscript.

\section{Funding}

This work was funded by the Natural Science Foundation of China (No. 82102012,82102006 ) and Natural Science Foundation of Jiangsu Province (No. BK20201118, BK20211008).

\section{Availability of data and materials}

Clinical, neuroimaging and statistical data will be available upon request from any qualified investigator.

\section{Declarations}

\section{Ethics approval and consent to participate}

All subjects underwent the same MRI scan and cognitive function assessment. The present study was approved by the local ethics of Nanjing Medical University. Written informed consent were obtained from all participants before undergoing MRI.

\section{Consent for publication}

Not applicable.

\section{Competing interests}

The authors declare that they have no competing interests.

\section{Author details}

'Department of Radiology, Nanjing First Hospital, Nanjing Medical University, No.68, Changle Road, 210006 Nanjing, China. ${ }^{2}$ Department of Rehabilitation Science, School of Public Health and Health Professions, University at Buffalo, Buffalo, USA.
Received: 1 August 2021 Accepted: 28 October 2021

Published online: 13 November 2021

\section{References}

1. Ashina H, Porreca F, Anderson T, Amin FM, Ashina M, Schytz HW et al (2019) Post-traumatic headache: epidemiology and pathophysiological insights. Nature reviews Neurology 15(10):607-617. doi:https://doi.org/10.1 038/s41582-019-0243-8

2. Lucas S, Hoffman JM, Bell KR, Walker W, Dikmen S (2012) Characterization of headache after traumatic brain injury. Cephalalgia: an international journal of headache 32(8):600-606. doi:https://doi.org/10.1177/0333102412445224

3. Howard L, Dumkrieger G, Chong CD, Ross K, Berisha V, Schwedt TJ (2018) Symptoms of Autonomic Dysfunction Among Those With Persistent Posttraumatic Headache Attributed to Mild Traumatic Brain Injury: A Comparison to Migraine and Healthy Controls. Headache 58(9):1397-1407

4. Váša F, Romero-Garcia R, Kitzbichler MG (2020) Conservative and disruptive modes of adolescent change in human brain functional connectivity. 117: 3248-3253. https://doi.org/10.1073/pnas.1906144117. 6

5. Zhu DC, Covassin T, Nogle S, Doyle S, Russell D, Pearson RL et al (2015) A potential biomarker in sports-related concussion: brain functional connectivity alteration of the default-mode network measured with longitudinal resting-state fMRI over thirty days. J Neurotrauma 32(5):327341. doi:https://doi.org/10.1089/neu.2014.3413

6. Dretsch MN, Rangaprakash D, Katz JS, Daniel TA, Goodman AM, Denney TS et al (2019) Strength and Temporal Variance of the Default Mode Network to Investigate Chronic Mild Traumatic Brain Injury in Service Members with Psychological Trauma. Journal of experimental neuroscience 13: 1179069519833966. doi:https://doi.org/10.1177/1179069519833966

7. Li F, Lu L, Shang S, Hu L, Chen H, Wang P et al (2020) Disrupted functional network connectivity predicts cognitive impairment after acute mild traumatic brain injury. 26:1083-1091. https://doi.org/10.1111/cns.13430. 10

8. Dumkrieger G, Chong CD, Ross K, Berisha V, Schwedt TJ (2019) Static and dynamic functional connectivity differences between migraine and persistent post-traumatic headache: A resting-state magnetic resonance imaging study. Cephalalgia: an international journal of headache 39(11): 1366-1381. doi:https://doi.org/10.1177/0333102419847728

9. Schwedt TJ, Chong CD, Peplinski J, Ross K, Berisha V (2017) Persistent posttraumatic headache vs. migraine: an MRI study demonstrating differences in brain structure. J Headache Pain 18(1):87. doi:https://doi.org/10.1186/s10194017-0796-0

10. Skorobogatykh K, van Hoogstraten WS, Degan D, Prischepa A, Savitskaya A, lleen BM et al (2019) Functional connectivity studies in migraine: what have we learned? J Headache Pain 20(1):108. doi:https://doi.org/10.1186/s10194019-1047-3

11. Lu L, Zhang J, Li F, Shang S, Chen H, Yin X et al (2021) Aberrant Static and Dynamic Functional Network Connectivity in Acute Mild Traumatic Brain Injury with Cognitive Impairment. Clin Neuroradiol 31(10):021-01082

12. Onton J, Makeig S (2006) Information-based modeling of event-related brain dynamics. Prog Brain Res 159:99-120

13. Rashid B, Damaraju E, Pearlson GD, Calhoun VD (2014) Dynamic connectivity states estimated from resting fMRI Identify differences among Schizophrenia, bipolar disorder, and healthy control subjects.Front Hum Neurosci. ; 8(897)

14. Allen EA, Damaraju E, Plis SM, Erhardt EB, Eichele T, Calhoun VD (1991) Tracking whole-brain connectivity dynamics in the resting state. Cerebral cortex (New York, NY: 2014;24(3):663-76. doi:https://doi.org/10.1093/cercor/ bhs352

15. Damaraju E, Allen EA, Belger A, Ford JM, McEwen S, Mathalon DH et al (2014) Dynamic functional connectivity analysis reveals transient states of dysconnectivity in schizophrenia. Neurolmage Clinical 5:298-308. doi:https:// doi.org/10.1016/j.nicl.2014.07.003

16. Fiorenzato E, Strafella AP, Kim J, Schifano R, Weis L, Antonini A et al (2019) Dynamic functional connectivity changes associated with dementia in Parkinson's disease. Brain 142(9):2860-2872. doi:https://doi.org/10.1093/bra in/awz192

17. Hou W, Sours Rhodes C, Jiang L, Roys S, Zhuo J, JaJa J et al (2019) Dynamic Functional Network Analysis in Mild Traumatic Brain Injury. Brain Connect 9(6):475-487. doi:https://doi.org/10.1089/brain.2018.0629

18. Vergara VM, Mayer AR, Kiehl KA, Calhoun VD (2018) Dynamic functional network connectivity discriminates mild traumatic brain injury through 
machine learning. Neurolmage Clinical 19:30-37. doi:https://doi.org/10.1016/ j.nicl.2018.03.017

19. Vergara VM, Mayer AR, Damaraju E, Calhoun VD (2017) The effect of preprocessing in dynamic functional network connectivity used to classify mild traumatic brain injury. Brain and behavior 7(10):e00809. doi:https://doi. org/10.1002/brb3.809

20. Carroll LJ, Cassidy JD, Holm L, Kraus J, Coronado VG (2004) Methodologica issues and research recommendations for mild traumatic brain injury: the WHO Collaborating Centre Task Force on Mild Traumatic Brain Injury. J Rehabil Med 43 Suppl113-125. doi:https://doi.org/10.1080/16501960410023 877

21. de Guise E, Alturki AY, LeBlanc J, Champoux MC, Couturier C, Lamoureux J et al (2014) The Montreal Cognitive Assessment in persons with traumatic brain injury. Applied neuropsychology Adult 21(2):128-135. doi:https://doi. org/10.1080/09084282.2013.778260

22. The International Classification of Headache Disorders, 3rd edition (beta version). Cephalalgia: an international journal of headache (2013);33(9):629808. doi:https://doi.org/10.1177/0333102413485658

23. Varndell W, Fry M, Elliott D (2017) A systematic review of observational pain assessment instruments for use with nonverbal intubated critically ill adult patients in the emergency department: an assessment of their suitability and psychometric properties. J Clin Nurs 26(1-2):7-32

24. Calhoun VD, Adali T, Pearlson GD, Pekar JJ (2001) A method for making group inferences from functional MRI data using independent component analysis. Hum Brain Mapp 14(3):140-151. doi:https://doi.org/10.1002/hbm.1 048

25. Erhardt EB, Rachakonda S, Bedrick EJ, Allen EA, Adali T, Calhoun VD (2011) Comparison of multi-subject ICA methods for analysis of fMRI data. Hum Brain Mapp 32(12):2075-2095. doi:https://doi.org/10.1002/hbm.21170

26. Li YO, Adali T, Calhoun VD (2007) Estimating the number of independent components for functional magnetic resonance imaging data. Hum Brain Mapp 28(11):1251-1266. doi:https://doi.org/10.1002/hbm.20359

27. Du Y, Pearlson GD, Yu Q, He H, Lin D, Sui J et al (2016) Interaction among subsystems within default mode network diminished in schizophrenia patients: A dynamic connectivity approach. Schizophr Res 170(1):55-65. doi: https://doi.org/10.1016/.jschres.2015.11.021

28. Malhi GS, Das P, Outhred T, Bryant RA, Calhoun V (2019) Resting-state neural network disturbances that underpin the emergence of emotional symptoms in adolescent girls: resting-state fMRI study. The British journal of psychiatry: the journal of mental science 215(3):545-551

29. Miller RL, Yaesoubi M, Turner JA, Mathalon D, Preda A, Pearlson G et al (2016) Higher Dimensional Meta-State Analysis Reveals Reduced Resting fMRI Connectivity Dynamism in Schizophrenia Patients. PLoS ONE 11(3): e0149849. doi:https://doi.org/10.1371/journal.pone.0149849

30. Veréb D, Szabó N, Tuka B, Tajti J, Király A, Faragó P et al (2020) Temporal instability of salience network activity in migraine with aura. Pain 161(4): 856-864

31. Legrain V, Damme SV, Eccleston C, Davis KD, Seminowicz DA, Crombez G (2009) A neurocognitive model of attention to pain: behavioral and neuroimaging evidence. Pain 144(3):230-2

32. Uddin LQ (2015) Salience processing and insular cortical function and dysfunction. Nat Rev Neurosci 16(1):55-61. doi.https://doi.org/10.1038/nrn3 857

33. Simons LE, Elman I, Borsook D (2014) Psychological processing in chronic pain: a neural systems approach. Neurosci Biobehav Rev 39:61-78

34. Raichle ME (2015) The brain's default mode network. Annu Rev Neurosci 38: 433-447

35. Nathan DE, Oakes TR, Yeh PH, French LM, Harper JF, Liu W et al (2015) Exploring variations in functional connectivity of the resting state default mode network in mild traumatic brain injury. Brain Connect 5(2):102-114. doi:https://doi.org/10.1089/brain.2014.0273

36. Niu X, Bai L (2019) Disruption of periaqueductal grey-default mode network functional connectivity predicts persistent post-traumatic headache in mild traumatic brain injury. 90:326-332. https://doi.org/10.1136/jnnp-2018-31 8886.3

37. Zou Y, Tang W, Qiao X, Li J (2021) Aberrant modulations of static functional connectivity and dynamic functional network connectivity in chronic migraine. Quant Imaging Med Surg 11(6):2253-2264

38. Diedrichsen J, King M, Hernandez-Castillo C, Sereno M, Ivry RB (2019) Universal Transform or Multiple Functionality? Understanding the
Contribution of the Human Cerebellum across Task Domains. Neuron 102(5):918-928. doi:https://doi.org/10.1016/..neuron.2019.04.021

39. Li F, Lu L, Shang S, Chen H, Wang P, Haidari NA et al (2020) Cerebral Blood Flow and Its Connectivity Deficits in Mild Traumatic Brain Injury at the Acute Stage.Neural plasticity. ; 1(2174371)

40. Hindriks R, Adhikari MH, Murayama Y, Ganzetti M, Mantini D, Logothetis NK et al (2016) Can sliding-window correlations reveal dynamic functional connectivity in resting-state fMRI? Neurolmage. 127:242-256. https://doi. org/10.1016/..neuroimage.2015.11.055

\section{Publisher's Note}

Springer Nature remains neutral with regard to jurisdictional claims in published maps and institutional affiliations.

\section{Ready to submit your research? Choose BMC and benefit from:}

- fast, convenient online submission

- thorough peer review by experienced researchers in your field

- rapid publication on acceptance

- support for research data, including large and complex data types

- gold Open Access which fosters wider collaboration and increased citations

- maximum visibility for your research: over $100 \mathrm{M}$ website views per year

At BMC, research is always in progress.

Learn more biomedcentral.com/submissions 